\title{
PERCEPCIÓN DEL BULLYING, GESTIÓN DE CONFLICTOS Y CLIMA ESCOLAR EN DIRECTIVOS Y DOCENTES DE CENTROS EDUCATIVOS
}

\author{
PERCEPTION OF BULLYING AND MANAGEMENT OF CONFLICT AND CLIMATE \\ IN SCHOOL OFFICERS AND TEACHERS SCHOOLS
}

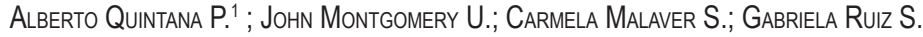 \\ Universidad Nacional Mayor de San Marcos, Lima, Perú \\ (RECIBIDO EL 16/08/2012 - ACEPTADO, 25/11/2012 )
}

\begin{abstract}
RESUMEN
Con el objetivo de explorar la relacion entre las habilidades de gestión para la negociación de conflictos, la percepción del bullying y el clima organizacional en la escuela que presentan los directivos y docentes de centros educativos, se investigó a los estamentos directivo y docente de seis centros educativos. La obtención de datos se hizo a través de tres instrumentos: el Cuestionario de Clima Social Organizacional y otros dos que fueron especialmente adaptados a nuestro medio: la escala de percepción de bullying en docentes y directivos de Centros Educativos y el Test de Habilidades para la Gestión en la Negociación de Conflictos. Se recogió la informacion mediante un diseño de tipo descriptivo-correlacional, con un muestreo no probabilístico circunstancial de la población a estudiar. Para cuantificar los resultados se aplicó el estadístico no paramétrico Tau de Kendall para establecer las correlaciones entre las variables mencionadas. Finalmente, se concluye que cuando en el clima organizacional escolar los directores que se muestran autocráticos se presenta mayor prevalencia de acoso entre pares en los centros educativos; mientras que, cuando en el clima organizacional escolar se presenta red permanente de amistad y apoyo entre el profesorado, va a presentarse menor prevalencia de acoso entre pares en los centros educativos.
\end{abstract}

Palabras claves: Percepción del bullying, gestión de conflictos, clima escolar, docentes

\begin{abstract}
In order to explore the relationship between management skills to negotiate conflicts, the perception of bullying and school organizational climate presented by principals and teachers of schools, was investigated for the estates management and teaching from six education. Data collection was done through three instruments: the Organizational Social Climate Questionnaire and two others that were specially adapted to our environment: the scale of perceived bullying teachers and administrators at Schools and Test Management Skills Negotiation in Conflict. Information was collected through a descriptive design and correlational, with a circumstantial non-probability sampling the study population. To quantify the results are applied the statistical nonparametric Kendall Tau to establish correlations between

1 Profesor Principal de la Facultad de Psicología de la UNMSM E-mail: albertolqp@gmail.com
\end{abstract}


these variables. Finally, we conclude that when the organizational climate school principals shown autocratic presented higher prevalence of peer harassment in schools, whereas, when the school organizational climate has permanent network of friendship and support among teachers, will present a lower prevalence of peer harassment in schools.

Keywords: Perception of bullying, conflict management, school climate, teacher

\section{INTRODUCCIÓN}

La agresión, intimidación o acoso entre escolares recibe la denominación universal de "bullying". Ésta es una de las formas de violencia que más repercusión está teniendo actualmente sobre las personas en edad escolar. En anteriores investigaciones (Quintana et al, 2009, 2010) trabajamos específicamente sobre alumnos espectadores de bullying cuya presencia refuerza o castiga este tipo de violencia, por lo que la investigación presente abordará el problema de otros aspectos del clima escolar, en relación con la conducta instrumental de solución de conflictos interpersonales en sus estamentos directivo y docente, para precisar su alcance propiciatorio de problemas de violencia entre pares y sugerir alternativas de gestión más adecuadas para evitarlos o disminuir su incidencia.

\section{Marco teórico, planteamiento de hipótesis}

El bullying, como un fenómeno sistémico, involucra en su afrontamiento a la comunidad educativa. Dentro de la dinámica de este tipo de violencia en la escuela; la tríada agresor, víctima, espectador no se circunscribe al grupo de pares; es fundamental para su visibilización y abordaje la participación activa, interactiva y cooperativa de docentes, orientadores, autoridades y la familia. Por lo cual el análisis del currículo oculto, de la cultura escolar hegemónica que subyace en las instituciones educativas, permite conocer sobre actitudes y creencias de los miembros de la comunidad educativa, acerca de la existencia, concepción, proceso e impacto que tiene este fenómeno de violencia entre pares en la convivencia escolar y de la injerencia de estas percepciones para manejar este tipo de conflictividad en la escuela orientando el cauce de la intervención ante este conflicto (Alzate, 1998), pudiendo ser esta de negociación, mediación, imposición, retirada o inactividad. Una forma de operacionalizarse es en el clima escolar visualizado como «el conjunto de características psicosociales de un centro educativo, determinadas por factores estructurales, personales y funcionales de la institución que, integrados en un proceso dinámico específico, confieren un peculiar estilo a dicho centro, condicionante, a la vez de los distintos procesos educativos» (Cere, 1993) esta atmósfera que se genere es gravitante para el fortalecimiento de una adecuada convivencia o bien para obstaculizarla, afectando su implementación a nivel organizativo, dirigencial, normativo, así como también para el logro de metas, funciones y procesos de comunicación, los cuales no deben tener un carácter excluyentemente informacional, instrumental enfocados a mantener la normatividad (Duarte, 2005), sino también, que incremente el nivel relacional y conlleve al establecimiento de vínculos afectivos, que coadyuven al desarrollo de 
patrones comportamentales de respeto y tolerancia. Por lo cual la evaluación y diagnóstico del clima escolar es importante para diseñar adecuadamente programas de intervención que permitan mejorar, en base a criterios objetivos y racionales, la eficiencia y eficacia de los centros educativos (Arévalo, 2002; Rodríguez, 2004). Al respecto uno de los problemas frecuentes en la interacción escolar, actualmente bajo la aguda observación de sectores especializados con el fin de prevenir tempranamente su aparición o impedir su mantenimiento, es el tema del bullying o violencia entre pares, fenómeno abordado por nosotros en anteriores investigaciones, en términos de manifestación agresiva infantil y juvenil en el ámbito escolar. Nuestro equipo viene trabajando específicamente en la línea de investigación del conflicto interpersonal englobado bajo el concepto de bullying adolescente, enfocado en la influencia del entorno social sobre este: el espectador de la violencia entre pares en sus diferentes modalidades (Avilés, 2006; Quintana, et al, 2009; 2010). En esta ocasión creemos indispensable complementar los resultados que venimos obteniendo en esos estudios anteriores explorando el "clima institucional", debido a que resulta plausible que los episodios de bullying se probabilicen propiciatoriamente a partir de climas organizacionales escolares donde prima una determinada orientación gestionaria. La obtención de datos en este rubro tiene dos ventajas: 1) permite ubicar qué estilo(s) de liderazgo (o gestión de conflictos entre directivos y docentes) se relacionaría(n) probablemente con la irresolución de problemas del bullying en las escuelas (Murillo y Becerra, 2009); y 2) coadyuvar a la autoevaluación del clima escolar para conocer sus debilidades y articular procedimientos gestionarios eficaces y efectivos (Malaret, 2001; Martínez y Codutti, 2008). En suma, se plantea conocer:

¿Cuál es la relación entre el bullying, el clima organizacional en la escuela y las habilidades de gestión para la negociación de conflictos en directivos y docentes?

Es necesario, por un lado, obtener información acerca de los modos de gestión directiva y docente relacionadas con la aparición y mantenimiento de los problemas de bullying, a fin de plantear soluciones efectivas; y por otro lado, afianzar la consistencia de los instrumentos de evaluación de ese fenómeno en nuestro medio, por lo cual utilizamos pruebas elaboradas o adaptadas a las condiciones socioculturales de nuestra realidad.

\section{Hipótesis}

Existe relación significativa entre las habilidades de gestión para la negociación de conflictos, la percepción del bullying y del clima organizacional en directivos $\mathrm{y}$ docentes de los centros educativos.

Cada dimensión de la gestión para la negociación de conflictos se relaciona significativamente con cada dimensión de la percepción del bullying en directivos y docentes de los centros educativos.

Cada dimensión de la gestión para la negociación de conflictos se relaciona significativamente con cada dimensión de la percepción del clima organizacional en directivos y docentes de los centros educativos. 
Cada dimensión de la percepción del bullying se relaciona significativamente con cada dimensión de la percepción del clima organizacional en directivos y docentes de los centros educativos

\section{MÉTODO}

En el presente trabajo han sido estudiadas las relaciones entre tres variables referidas a procesos psicológicos de los directivos y docentes de instituciones educativas en nuestro medio: Percepción de bullying, habilidades para la gestión de negociación de conflictos y el Clima Organizacional que perciben en la escuela. Habiéndose a su vez controlado el impacto de las variables: Nivel socio-económico (C), Instrucción (Secundaria), Sexo (ambos) y Área geográfica (Lima Metropolitana).

El estudio se realizó mediante un diseño de investigación de tipo descriptivo correlacional, en una muestra de seis centros educativos pertenecientes a los niveles socioeconómicos $\mathrm{C}$ y $\mathrm{D}$ de la población de Lima, seleccionados mediante un muestreo de tipo no probabilístico, circunstancial. Es susceptible de esta tipificación, en tanto, en la muestra se evalúan dos o más variables en las cuales se analizan relaciones de influencia en magnitud y dirección. Es decir, se evalúa si el crecimiento o disminución del valor de una de ellas está asociada al aumento o disminución en el valor de otra, no pudiendo en consecuencia hacer afirmaciones causales, sino solo predecir el comportamiento de una variable a partir de la observación del comportamiento de la otra.

Para ello han sido administrados a la muestra los siguientes instrumentos de recolección de datos:

Ficha demográfica: Permite registrar algunas características relevantes de los sujetos estudiados: Institución de procedencia, Edad, Sexo, Tiempo de permanencia en la institución, etc.

Test de Habilidades para la Gestión en la Negociación de Conflictos: (Adaptada y validada a partir de la prueba de Vicuña, Hernández, Paredes y Ríos, 2008). Cuenta con 50 ítems de respuesta tipo lickert que mide las habilidades de comunicación, compromiso, cognición interpersonal, control emocional y empatía. Para el presente estudio se obtuvo la validez por criterio de expertos con un Coeficiente Aiken de 0.85 y una confiabilidad con el coeficiente Alfa de Cronbach de .833 . Las cinco habilidades para la gestión de conflictos evaluadas son las siguientes: Comunicación, Capacidad para usar eficazmente herramientas de comunicación verbal y no verbal, que se expresan en cómo se escucha, cómo se responde gestual, posturalmente y verbalmente; Compromiso, Capacidad para involucrarse con el conflicto, en el sentido de sentirse cómodo participando en su solución, identificándose con el papel de negociador; Cognición Interpersonal, Capacidad de conocer las causas, generar alternativas de solución y planificar su ejecución, considerando las consecuencias en los participantes en conflictos interpersonales; Control emocional, Capacidad para usar sus recursos personales o potenciales para evitar que la carga emocional que le llega, termine por bloquearlo emocio- 
nalmente, irritándolo o asumiendo posturas preferenciales, o estresándolo, y por ende disminuyendo su eficiencia y eficacia negociadora; y, Empatía, Capacidad para abandonar sus puntos de vista y asumir en cada instante los puntos de vista de la persona o las personas en conflicto.

Escala de Percepción de bullying en docentes y directivos de Centros Educativos: elaborada y validada por el responsable y su equipo de investigadores a partir de un análisis crítico del Cuestionario sobre Preconcepciones de Intimidación y Maltrato Entre Iguales (Adaptado de Ortega, Mora-Merchán y Mora-Fernández), complementado con los aportes de Monjas y Avilés, 2003). Cuenta con 24 ítems de respuesta tipo lickert. Para el presente estudio se obtuvo la validez por criterio de expertos con un Coeficiente Aiken de 0.80 y una confiabilidad con el coeficiente Alfa de Cronbach de .645. Este instrumento permite dar cuenta de las dimensiones: Información: La información específica que posee el docente o directivo respecto a la violencia entre pares o bullying; Sensibilización: Capacidad para identificar y conmoverse frente a la violencia entre pares; Capacidad Afronte: Autoconcepto de la propia capacidad para actuar de forma preventiva ante la violencia entre pares; Percepción del Bullying: Magnitud de la violencia entre pares que el docente o directivo evidencia en la institución educativa; y, Percepción de Acoso a los docentes: Magnitud de la violencia de los alumnos hacia el docente que se evidencia en la institución educativa.

Cuestionario Descriptivo del Clima Organizacional, OCDQ-RE: (Organizational climate description questionnaire) de Halpin y Croft (1983), es un instrumento que consta de 42 ítems y que resume el clima organizativo en seis dimensiones, refiriéndose tres de ellas al comportamiento del director y las otras tres en relación con el comportamiento del profesorado. En el presente estudio se obtuvo la validez por criterio de expertos con un Coeficiente Aiken de 0.90 y una confiabilidad con el coeficiente Alfa de Cronbach de .833. Las dimensiones que el instrumento evalúa acerca de la forma en que se percibe el clima en referencia a los directivos: Apoyo, La conducta de apoyo del director se refleja en sus buenas relaciones con el profesorado. Estos directores respetan la competencia profesional de los profesores e intentan mostrar su interés personal por cada uno. Disfrutan trabajando con los profesores en la fijación de metas, la resolución de problemas y aceptan de buen grado las sugerencias y las opiniones de estos. Expresan alabanzas con sinceridad y con frecuencia y utilizan la crítica de forma constructiva; Directividad, La conducta directiva la manifiestan aquellos directores que se muestran rígidos y mantienen la distancia con los profesores. Estos directores necesitan mantener un estrecho y constante control sobre todos los profesores y actividades de la escuela, hasta en los detalles más pequeños. Estos directores son autocráticos y no prestan consideración a las relaciones personales; y, Restrictividad, La conducta restrictiva la manifiestan aquellos directores que desalientan las relaciones y las iniciativas del profesorado porque su única preocupación se refiere al estricto cumplimiento de las normas, los procedimientos y los detalles administrativos. Los directores restrictivos no dejan sitio a las iniciativas y agobian a los demás con actividades no educativas. Las dimensiones que el instrumento evalúa acerca la forma en que 
se percibe el clima organizacional en referencia a los docentes: Profesionalidad, La conducta profesional está caracterizada por las relaciones de apoyo mutuo y basadas en el propio trabajo por parte de los profesores. Estos se sienten orgullosos de su escuela, disfrutan trabajando con sus colegas y se guían por el sentimiento de realización y satisfacción en el trabajo. Muestran energía, entusiasmo y optimismo; Relaciones sociales, Esta categoría refleja una red permanente de apoyo entre el profesorado. Estos llegan a conocerse bien entre sí hasta el punto de establecer relaciones de amistad y relacionarse regularmente tanto dentro como fuera del entorno escolar; y, Despreocupación. La conducta de despreocupación se presenta en aquellos profesores que no se implican verdaderamente en la escuela, con sus compañeros y su profesión. Se encuentran simplemente pasando el tiempo y son elementos improductivos para las tareas grupales y de equipo; no contribuyen a los objetivos comunes. Su conducta es negativa y crítica hacia sus colegas y hacia la organización.

Para analizar los resultados se, elaboró una base de datos en una hoja de cálculo de la aplicación Excel y, luego con el software SPSS versión .16 se contrastaron las hipótesis enunciadas, procesando estos datos con el estadístico no paramétrico Tau de Kendall, a fin de establecer las correlaciones previstas en estas hipótesis entre las variables correspondientes.

\section{Exposición de resultados y validación de hipótesis}

Resultados encontrados respecto a la Hipótesis 2, cada dimensión de las habilidades de gestión para la negociación de conflictos se relaciona significativamente con cada dimensión de la percepción del bullying en directivos y docentes de los centros educativos.

Tabla 1. Correlación entre cada dimensión de las habilidades de gestión para la negociación de conflictos y cada dimensión de la percepción del bullying en directivos y docentes de los centros educativos.

\begin{tabular}{|c|c|c|c|c|c|c|}
\hline & & $\begin{array}{l}\text { COMUNI } \\
\text { CACIÓN }\end{array}$ & COGNICIÓN & $\begin{array}{l}\text { COMPRO } \\
\text { MISO }\end{array}$ & CONTROL & EMPATÍA \\
\hline \multirow{2}{*}{ SENSIBILIZACIÓN } & \multirow{2}{*}{$\begin{array}{l}\text { Coeficiente de } \\
\text { correlación } \\
\text { Sig. (bilateral) } \\
\mathrm{N}\end{array}$} & -169 & -174 & .139 & .083 & .231 \\
\hline & & $\begin{array}{l}.236 \\
146\end{array}$ & $\begin{array}{l}.228 \\
145\end{array}$ & $\begin{array}{r}.339 \\
146\end{array}$ & $\begin{array}{r}.570 \\
145\end{array}$ & $\begin{array}{r}.102 \\
146\end{array}$ \\
\hline \multirow{2}{*}{ AFRONTE } & \multirow{2}{*}{$\begin{array}{l}\text { Coeficiente de } \\
\text { correlación } \\
\text { Sig. (bilateral) } \\
\text { N }\end{array}$} & .047 & -185 & -133 & .077 & -.174 \\
\hline & & $\begin{array}{l}.742 \\
145\end{array}$ & $\begin{array}{r}.198 \\
144\end{array}$ & $\begin{array}{r}.354 \\
145\end{array}$ & $\begin{array}{l}.596 \\
144\end{array}$ & $\begin{array}{l}.213 \\
145\end{array}$ \\
\hline \multirow{2}{*}{ INFORMACIÓN } & \multirow{2}{*}{$\begin{array}{l}\text { Coeficiente de } \\
\text { correlación } \\
\text { Sig. (bilateral) } \\
\text { N }\end{array}$} & 177 & .172 & -105 & .124 & -.184 \\
\hline & & $\begin{array}{l}.212 \\
146\end{array}$ & $\begin{array}{l}.230 \\
145\end{array}$ & $\begin{array}{l}.461 \\
146\end{array}$ & $\begin{array}{l}.392 \\
145\end{array}$ & $\begin{array}{l}.188 \\
146\end{array}$ \\
\hline \multirow[b]{2}{*}{ ACOSO_ENTRE_PARES } & \multirow{2}{*}{$\begin{array}{l}\text { Coeficiente de } \\
\text { correlación } \\
\text { Sig. (bilateral) } \\
\text { N }\end{array}$} & .144 & .043 & $\left.-.312^{*}\right)$ & -.312 & -.324 \\
\hline & & .312 & .763 & .031 & .366 & .022 \\
\hline \multirow{3}{*}{ ACOSO_AL_DOCENTES } & \multirow{3}{*}{$\begin{array}{l}\text { Coeficiente de } \\
\text { correlación } \\
\text { Sig. (bilateral) } \\
\text { N }\end{array}$} & -105 & $\begin{array}{l}144 \\
.085\end{array}$ & $\begin{array}{r}145 \\
-.185\end{array}$ & $\begin{array}{r}.144 \\
-.185\end{array}$ & $\begin{array}{r}145 \\
-.142\end{array}$ \\
\hline & & .464 & .560 & .199 & .413 & .310 \\
\hline & & 146 & 145 & 147 & 145 & 145 \\
\hline
\end{tabular}

* La correlación es significativa al nivel 0,05 (bilateral). 
Por los resultados de la Tabla 1, podríamos concluir que en este caso se acepta la hipótesis nula que no habría relación entre las dimensiones de ambos procesos, a excepción de la propia dimensión de Acoso entre pares de la variable percepción del bullying (que mide propiamente el núcleo semántico del referido proceso) la cual evidencia una correlación inversa y significativa moderada (al 0.05) con las dimensiones de compromiso y empatía de las habilidades de gestión para la negociación de conflictos que muestren directivos y docentes de los centros educativos.

Resultados observados respecto a la Hipótesis 3. Cada dimensión de las habilidades de gestión para la negociación de conflictos se relaciona significativamente con cada dimensión de la percepción del clima organizacional escolar que muestran los directivos y docentes de los centros educativos.

Tabla 2. Correlación entre cada dimensión de las habilidades de gestión para la negociación de conflictos y cada dimensión de la percepción del clima organizacional escolar en directivos y docentes de los centros educativos.

\begin{tabular}{|c|c|c|c|c|c|c|c|}
\hline & & $\begin{array}{l}\text { RELACIONES } \\
\text { _SOCIALES }\end{array}$ & $\begin{array}{l}\text { RESTRIC- } \\
\text { TIVIDAD }\end{array}$ & $\begin{array}{l}\text { DIRECTI- } \\
\text { VIDAD }\end{array}$ & APOYO & $\begin{array}{l}\text { PROFESIO- } \\
\text { NALISMO }\end{array}$ & $\begin{array}{l}\text { DESPREOCU- } \\
\text { PACIÓN }\end{array}$ \\
\hline \multirow{3}{*}{ COMUNICACIÓN } & \multirow{3}{*}{$\begin{array}{l}\text { Coeficiente de } \\
\text { correlación } \\
\text { sig. (bilateral) } \\
\mathrm{N}\end{array}$} & .254 & $.315\left(^{*}\right)$ & .111 & .154 & .202 & .075 \\
\hline & & .085 & .027 & .414 & .266 & .149 & .596 \\
\hline & & 146 & 149 & 149 & 148 & 149 & 147 \\
\hline \multirow{3}{*}{ COGNICIÓN } & \multirow{3}{*}{$\begin{array}{l}\text { Coeficiente de } \\
\text { correlación } \\
\text { sig. (bilateral) } \\
\mathrm{N}\end{array}$} & $.315(*)$ & .057 & $.424\left(^{*}\right)$ & .141 & .176 & .101 \\
\hline & & .037 & .692 & .002 & .316 & .213 & .474 \\
\hline & & 145 & 148 & 148 & 147 & 148 & 147 \\
\hline \multirow{3}{*}{ COMPROMISO } & \multirow{3}{*}{$\begin{array}{l}\text { Coeficiente de } \\
\text { correlación } \\
\text { sig. (bilateral) } \\
\mathrm{N}\end{array}$} & .245 & -.132 & $.340(*)$ & .060 & .030 & .183 \\
\hline & & .100 & .362 & .014 & .670 & .832 & .203 \\
\hline & & 146 & 149 & 149 & 148 & 149 & 146 \\
\hline \multirow{3}{*}{ CONTROL } & \multirow{3}{*}{$\begin{array}{l}\text { Coeficiente de } \\
\text { correlación } \\
\text { sig. (bilateral) } \\
\mathrm{N}\end{array}$} & .288 & -.005 & $.277\left(^{*}\right)$ & .000 & .129 & .112 \\
\hline & & .056 & .971 & .045 & 1.000 & .370 & .441 \\
\hline & & 145 & 148 & 148 & 147 & 148 & 145 \\
\hline \multirow{3}{*}{ EMPATIA } & \multirow{3}{*}{$\begin{array}{l}\text { Coeficiente de } \\
\text { correlación } \\
\text { sig. (bilateral) } \\
N\end{array}$} & .139 & -.122 & .160 & .106 & -217 & -.131 \\
\hline & & .341 & .387 & .232 & .437 & .116 & .349 \\
\hline & & 146 & 148 & 149 & .148 & 149 & 146 \\
\hline
\end{tabular}

* La correlación es significativa al nivel 0,05 (bilateral).

** La correlación es significativa al nivel 0,01 (bilateral).

De los resultados evidenciados en la Tabla 2, podríamos concluir que en este caso se acepta parcialmente la hipótesis alternativa, en el sentido que habría una relación estadísticamente significativa entre las dimensiones de ambos procesos, ya que cuatro de las cinco dimensiones habilidades de gestión para la negociación de conflictos que muestran los directivos y docentes de los centros educativos, evidencian moderadas correlaciones positivas estadísticamente significativas con tres de las seis dimensiones de la percepción del clima organizacional escolar que poseen. 
Resultados observados respecto a la Hipótesis 4. Cada dimensión de la percepción del bullying se relaciona significativamente con cada dimensión de la percepción del clima organizacional escolar en los directivos y docentes de los centros educativos.

Tabla 3. Correlacion entre cada dimensión de la percepción del bullying y cada dimensión de la percepción del clima organizacional escolar en directivos y docentes de los centros educativos.

\begin{tabular}{|c|c|c|c|c|c|c|c|}
\hline & & $\begin{array}{l}\text { DESPREOCU- } \\
\text { PACIÓN }\end{array}$ & $\begin{array}{c}\text { RELACIONES } \\
\text { _SOCIALES }\end{array}$ & $\begin{array}{l}\text { RESTRIC- } \\
\text { TIVIDAD }\end{array}$ & $\begin{array}{l}\text { DIRECTI- } \\
\text { VIDAD }\end{array}$ & APOYO & $\begin{array}{l}\text { PROFESIO- } \\
\text { NALISMO }\end{array}$ \\
\hline \multirow{3}{*}{ SENSIBILIZACÓN } & \multirow{3}{*}{$\begin{array}{l}\text { Coeficiente de } \\
\text { correlación } \\
\text { Sig. (bilateral) } \\
\text { N }\end{array}$} & .030 & .102 & .187 & $.291\left(^{*}\right)$ & $.315\left(^{*}\right)$ & -.002 \\
\hline & & .830 & .476 & .177 & .028 & .019 & .989 \\
\hline & & 147 & 147 & .150 & 150 & 149 & 150 \\
\hline \multirow{3}{*}{ AFRONTE } & \multirow{3}{*}{$\begin{array}{l}\text { Coeficiente de } \\
\text { correlación } \\
\text { Sig. (bilateral) } \\
\text { N }\end{array}$} & -.027 & .064 & .055 & .070 & .184 & -.004 \\
\hline & & .845 & .645 & .688 & .595 & .166 & .979 \\
\hline & & 146 & 146 & 149 & 149 & 148 & 150 \\
\hline \multirow{3}{*}{ INFORMACIÓN } & \multirow{3}{*}{$\begin{array}{l}\text { Coeficiente de } \\
\text { correlación } \\
\text { Sig. (bilateral) } \\
\text { N }\end{array}$} & .076 & .009 & .148 & .166 & .039 & .144 \\
\hline & & .578 & .948 & .283 & .206 & .769 & .285 \\
\hline & & 147 & 147 & 150 & 150 & 149 & 150 \\
\hline \multirow{3}{*}{ ACOSO_ENTRE_PARES } & \multirow{3}{*}{$\begin{array}{l}\text { Coeficiente de } \\
\text { correlación } \\
\text { Sig. (bilateral) } \\
\text { N }\end{array}$} & -.198 & $-.383\left(^{*}\right)$ & $295(*)$ & -.017 & .012 & -.037 \\
\hline & & .148 & .007 & .033 & .896 & .926 & .786 \\
\hline & & 146 & 146 & 149 & 149 & 148 & 149 \\
\hline \multirow{3}{*}{ INFORMACIÓN } & \multirow{3}{*}{$\begin{array}{l}\text { Coeficiente de } \\
\text { correlación } \\
\text { Sig. (bilateral) } \\
\text { N }\end{array}$} & -.095 & -.007 & .133 & .000 & .091 & -.138 \\
\hline & & .494 & .052 & .341 & 1.000 & .504 & .313 \\
\hline & & 147 & 147 & 150 & 150 & 149 & 150 \\
\hline
\end{tabular}

* La correlación es significativa al nivel 0,05 (bilateral).

** La correlación es significativa al nivel 0,01 (bilateral).

De los resultados evidenciados en la Tabla 3, podríamos concluir que en este caso nuevamente se acepta parcialmente la hipótesis alternativa, en el sentido que habría una relación estadísticamente significativa entre las dimensiones de ambos procesos, ya que cuatro de las seis dimensiones de la percepción del clima organizacional escolar que muestran los directivos y docentes de los centros educativos, evidencian moderadas a bajas correlaciones positivas estadísticamente significativas con dos de las cinco dimensiones de la percepción del bullying de la que dan cuenta, una de las cuales es la dimensión de acoso entre pares, que como explicitamos líneas arriba mide propiamente el núcleo semántico del referido proceso.

De estos resultados referidos a las hipótesis específicas, podemos concluir que la hipótesis general postulada, hipótesis 1 , acerca de la existencia de relaciones significativas entre las habilidades de gestión para la negociación de conflictos, 
la percepción del bullying y del clima organizacional en directivos y docentes de los centros educativos, en suma, estaría parcialmente comprobada.

\section{Análisis y sistematización}

Analizando el detalle de los resultados mostrados en la Tabla 1, encontramos que si bien los valores del coeficiente de correlación positiva entre las dimensiones de percepción de bullying en directivos y docentes, y las habilidades de gestión para la negociación de conflictos que ellos poseen, no llegan a ser estadísticamente significativos, sin embargo, la dimensión acoso entre pares muestra moderadas correlaciones inversas y significativas con las habilidades de gestión de conflictos compromiso (-.312) y empatía (-.324), lo cual indicaría que cuando los docentes o directivos muestran más habilidades empáticas y un mayor compromiso se presentan menores niveles de acoso entre pares en sus centros educativos. Valdría decir que en la medida que los docentes y directivos se involucran con los conflictos de los alumnos, y participan en su solución, analizando estos problemas interpersonales desde los puntos de vista de los interesados, como negociadores hábiles y eficaces, disminuye la prevalencia del acoso entre pares, bullying, en las instituciones de las cuales forman parte.

Examinando los resultados mostrados en la Tabla 2, encontramos que se producen relaciones positivas moderadas y estadísticamente significativas entre la habilidad de gestión para la negociación de conflictos cognición interpersonal con la dimensión relaciones sociales (.315) de la percepción del clima organizacional escolar que muestran los directivos y docentes de los centros educativos, presentándose también una correlación moderada entre la habilidad de comunicación y la dimensión restrictividad (.424) del clima organizacional escolar, así mismo, encontramos correlaciones significativas moderadas entre las habilidades de gestión para la negociación de conflictos cognición interpersonal (.424), compromiso (.340) y control (.277) con la dimensión directividad de la percepción del clima organizacional escolar que muestran los directivos y docentes de los centros educativos. Siendo que la única habilidad de gestión para la negociación de conflictos que no muestra una correlación significativa con alguna de las dimensiones de la percepción del clima organizacional escolar es la habilidad de empatía. Este análisis permite la interpretación de que los docentes y directivos estarían en mejores condiciones de generar y ejecutar alternativas de solución, considerando las consecuencias de las mismas en los alumnos participantes en conflictos interpersonales, en tanto más involucrados estén con las personas de su entorno escolar (colegas, directivos, administrativos y alumnos). Además que los docentes que muestran la habilidad de comunicarse gestual, postural y verbalmente, y a escuchar con mayor eficacia, van a tender a percibir a los directores como desalentadores de sus iniciativas y agobiantemente preocupados por el estricto cumplimiento de las normas, los procedimientos y los detalles administrativos, vale decir tienden a percibir a sus directores como burocráticos. Por último, a medida que los docentes tienden a presentar una mayor habilidad para generar y ejecutar alternativas de solución, considerando las consecuencias de las mismas en los alumnos participantes en 
conflictos interpersonales, involucrándose en el rol de negociadores en los mismos, y a ser emocionalmente más controlados, dando solución por sí mismos a los problemas interpersonales del alumnado, a la vez tienden a percibir a los directores como más rígidos, emocionalmente distantes de los profesores y alumnado, desarrollando un estrecho y constante control sobre todos ellos y todas las actividades de la escuela, hasta en los detalles más pequeños, es decir, tienden a percibir a sus directores como autocráticos.

La revisión en detalle de los resultados de la tabla 3 evidencia una correlación moderada y positiva entre las variables percepción de bullying y clima organizacional escolar en relación a la dimensión sensibilización del primero con las dimensiones directividad (.291) y apoyo (.315) del segundo. Se observa también esta misma correlación entre las dimensiones acoso entre pares y restrictividad (.295), Por otra parte hallamos una correlación moderada y negativa entre acoso entre pares y relaciones sociales (.383). Estos resultados permiten inferir que tanto los docentes que perciben que su director se muestra rígido, manteniéndose emocionalmente distante, de los profesores, y desarrollando un estrecho y constante control sobre todos ellos y todas las actividades de la escuela, hasta en los detalles más pequeños, como aquellos docentes que perciben que su director respeta su competencia profesional e intenta mostrar su interés personal por cada uno y disfruta trabajando con los profesores en la fijación de metas, la resolución de problemas y acepta de buen grado las sugerencias y las opiniones de estos, van a mostrarse sensibles a identificar y conmoverse frente a los episodios de maltrato entre pares de los alumnos, vale decir, la capacidad para identificar y conmoverse frente a los episodios de la violencia entre pares, de los docentes, no va a ser afectada por el tipo de liderazgo que perciben en los directivos. De otro lado, cuando en el clima organizacional escolar, los directivos desalientan las iniciativas de los docentes y los agobian preocupados por el estricto cumplimiento de las normas, los procedimientos y los detalles administrativos, se van a reportar también más frecuentes episodios de maltrato entre pares, bullying, en la institución educativa, mientras que por el contrario, cuando en el ambiente organizacional escolar, los docentes llegan a conocerse bien entre sí hasta el punto de establecer relaciones de amistad y relacionarse regularmente tanto dentro como fuera del entorno escolar, y sienten que cuentan con una red de apoyo entre ellos, van reportar que los episodios de maltrato entre pares, bullying, son menos frecuentes.

De otro lado, y complementariamente a la contrastación de las hipótesis de nuestro estudio, se han encontrado correlaciones significativas (de .266 a .409) entre las dimensiones del instrumento adaptado para medir percepción del bullying, las cuales evidencian que estarían evaluando el mismo proceso, aunque por la limitada cantidad de sujetos de la muestra no ha resultado pertinente realizar un análisis factorial de estos datos a fin de validar empíricamente las citadas dimensiones. De igual manera se han encontrado, al igual que en otros estudios, correlaciones significativas (de .296 a .574) entre las dimensiones del instrumento utilizado para evaluar la percepción del clima organizacional en directivos y docentes de los centros educativos. Por último, se han encontrado correlaciones significativas (de .361 
a .425) entre las dimensiones del instrumento que con el equipo de investigación adaptamos en un estudio anterior para medir las habilidades de gestión para la negociación de conflictos, las cuales también evidencian que estarían evaluando el mismo proceso.

\section{DISCUSIÓN}

La capacidad para identificar y conmoverse frente a los episodios de la violencia entre pares no va a ser afectada por el tipo de liderazgo que en el clima organizacional escolar perciben de los docentes en los directivos.

Cuando en el clima organizacional escolar los directores que se muestran rígidos y mantienen la distancia con los profesores, teniendo un estrecho y constante control sobre todos los profesores y actividades de la escuela, hasta en los detalles más pequeño, son considerados autocráticos y se presenta mayor prevalencia de acoso entre pares en los centros educativos. Conclusión que coincide con lo afirmado por Murillo y Becerra (2009) en el sentido que el(los) estilo(s) de liderazgo (o gestión de conflictos entre directivos y docentes) se relacionaría(n) probablemente con la irresolución de problemas del bullying en las escuelas.

Cuando en el clima organizacional escolar se establece una red permanente de apoyo entre el profesorado. Los docentes llegan a conocerse bien entre sí hasta el punto de establecer relaciones de amistad y relacionarse regularmente tanto dentro como fuera del entorno escolar. Van a evidenciarse más habilidades para la gestión de conflictos en los docentes y va a presentarse menor prevalencia de acoso entre pares en los centros educativos. Coincidiendo con los postulados de Duarte (2005), cuando afirmaba que en los climas organizacionales de las escuelas además del logro de metas, funciones y procesos de comunicación, enfocados a mantener la normatividad, sería conveniente que se incremente el nivel relacional entre los miembros conllevando al establecimiento de vínculos afectivos, que coadyuven al desarrollo de patrones comportamentales de respeto y tolerancia.

Los docentes que cuentan con mayores habilidades para la gestión de conflictos van a tender a percibir a los directores como más rígidos y manteniendo distancia emocional con los profesores y un estrecho y constante control sobre todas actividades de la escuela, hasta en los detalles más pequeños, considerándolos autocráticos, o bien, como prioritariamente preocupados solo por el estricto cumplimiento de las normas, los procedimientos y los detalles administrativos, no dejando lugar a las iniciativas y agobiándolos con actividades no educativas; es decir, desalentando las relaciones y las iniciativas del profesorado. Es pertinente explicitar que en tanto una limitación del presente estudio ha sido la naturaleza correlacional del mismo que no permite extraer nexos de causalidad entre los procesos, en esta última conclusión hemos asumido que las habilidades para la gestión de conflictos de los docentes son las que explican la percepción del clima organizacional escolar que estos tienen, y no que estas habilidades sean causadas por la percepción del clima organizacional como en las conclusiones 
anteriores hemos hecho, por ser esta última hipótesis menos plausible, puesto que se conoce que por el contrario, el énfasis en el cumplimiento de las leyes impulso de la organización y códigos y procedimientos para la estructura formal, estándar, inhibe a la gente a practicar los actos espontáneos (Rego, Arménio 2002), sin embargo, siendo la psicología una ciencia fáctica es menester realizar nuevas investigaciones con los controles observacionales necesarios a fin de someter a contrastación la presente conclusión.

\section{CONCLUSIONES}

Es conveniente resaltar, que los alcances del presente estudio en lo mediato serán articular o auspiciar estrategias de promoción, prevención, asistencia y rehabilitación de comportamiento pro-social en la población adolescente afectada, mediante el desarrollo de habilidades de gestión para la negociación de conflictos y el fomento de un clima escolar de bienestar en directivos y docentes de los centros educativos, mediante el desarrollo de actividades de perfeccionamiento profesional en relación con el tema investigado y la propuesta de programas de intervención para el desarrollo de Comunidades Educativas resilientes, vale decir, coincidiendo con Arévalo (2002) y Rodríguez (2004), diseñar programas de intervención que permitan mejorar, en base a criterios objetivos y racionales, la eficiencia y eficacia de los centros educativos.

\section{REFERENCIAS BIBLIOGRÁFICAS}

Alzate, R. (1998): Análisis y resolución de conflictos. Una perspectiva psicológica, Bilbao, Universidad del País Vasco.

Arévalo, E.E. (2002). Clima escolar y niveles de interacción social en estudiantes de secundarios del Colegio Claretiano de Trujillo. Tesis de Maestría en Psicología Educativa. Facultad de Psicología de la Universidad Nacional Mayor de San Marcos. Lima.

Avilés, J. (2006). Bullying: El maltrato entre iguales. Salamanca: Amaru.

Cere (1993): «Evaluar el contexto educativo». Documento de Estudio. Vitoria: Ministerio de Educación y Cultura, Gobierno Vasco.

Duarte, J. (2005). Violencia en la escuela. Comunicación y convivencia escolar en la cuidad de Medellín. OEI. Revista Iberoamericana de Educación, 37.

I.E.S. La Ería. (2008) Cuestionario sobre Preconcepciones de Intimidación y Maltrato Entre Iguales (Adaptado de Ortega, Mora-Merchán y Mora-Fernández); recuperado de http://www.deciencias.net/convivir/cuestionarios/alum_maltrato/6.Preconcimeimaltrato(Aviles).pdf

Martínez, D. F. y Codutti, T.N. (2008). Mediación escolar. violencia, autoevaluación y gestión de conflictos. Recuperado de: http://portal.educ.ar/debates/eid/docenteshoy/ mediacion-escolar/mediacion-escolar-violencia-au.php

Malaret, J. (2001). Manual de negociación y mediación. Madrid: Colex. 
Monjas, M. y Aviles, J. (2003) Programa de Sensibilización contra el maltrato entre iguales. Junta de Castilla y Leon: Valladoli

Murillo, P. y Becerra, S. (2009). Las percepciones del clima escolar por directivos, docentes y alumnado mediante el empleo de «redes semánticas naturales». Su importancia en la gestión de los centros educativos. Revista de Educación, 350, 375-399.

Ponferrada, M. (2008). TÍTOL: Climas escolares, malestares y relaciones entre iguales en las escuelas catalanas de secundaria. REVISTA D'ESTUDIS DE LA VIOLÈNCIA Nùm. 4, enero / marzo, recuperado de http://www.icev.cat/articulo_bullying_UAB. pdf

Quintana, A., Montgomery, W. y Malaver, C. (2009). Modos de afrontamiento y conducta resiliente en adolescentes espectadores de violencia entre pares. Revista IIPSI, 12(1), 153-171.

Quintana, A., Montgomery, W., Malaver, C. y Ruiz, G. (2010). Capacidad de disfrute y percepción del apoyo comunitario en espectadores de episodios de violencia entre pares. Revista IIPSI,13(1), 139-162.

Rego, Arménio (2002) Climas Éticos e Comportamentos de Cidadania Organizacional Revista de Administração de Empresas. v. 42. No 1.

Rodríguez, N. (2004). El clima escolar. Revista Digital Investigación y Educación 7(3), recuperado de http://www.csicsif.es/andalucia/modules/mod_sevilla/archivos/ revistaense/n7v3/clima.PDF.

Vicuña, L. Hernández, H., Paredes, M. y Ríos, J. (2008). Elaboración del Test de Habilidades para la Gestión en Negociación de Conflictos. Revista IIPSI, 11(2), 183-200. 